\title{
Load-Balancing Channel Assignment Algorithms for a Multi-Radio Multi-Channel Wireless Mesh Networks
}

\author{
Liang $\mathrm{Li}^{1}$, Siyuan Zhang ${ }^{1}$, Xiongwen Zhao ${ }^{1, *}$, Yi Ding ${ }^{2}$ and Junyu Liu ${ }^{2}$ \\ ${ }^{1}$ School of Electrical and Electronic Engineering, North China Electric Power University, Beijing, 102206, China. \\ ${ }^{2}$ Guodiantong Corporation, State Grid Information \& Telecommunications Group, Beijing, 100070, China \\ ${ }^{*}$ Corresponding author
}

\begin{abstract}
Capacity in multi-radio multi-channel wireless mesh networks can be greatly enhanced by adopting effective load balancing algorithms. In this work, a new channel allocation algorithm is proposed by considering inter-channel interference and load balance issue to maximize network capacity. The algorithm can choose a best channel based on channel interference caused by neighbor nodes and then to allocate the channel to a link so that it can bear more traffic and reduce network congestion. By comparison with the other two typical channel allocation algorithms available, it's found that our proposed algorithm has better network performance in case of heavy network load.
\end{abstract}

Keywords-wireless mesh network; channel assignment; maximizing capacity; load-balancing

\section{INTRODUCTION}

Wireless mesh network (WMN) is a promising network to provide users with flexible wireless connection in internet of things (IoT), in which using multi-radio multi-channel (MRMC) technology is one of the best solutions to support higher data transmission and improve network performance [1]. So far, lots of research on MRMC have been carried out in worldwide. In [2], a centralized channel assignment scheme including a spanner-based topology control and a link-ranking channel assignment algorithm was proposed by ranking the links based on balancing traffic loads. In [3], by taking into account their difference in traffic between the nodes, a distributed load sharing channel allocation algorithm (LBCA) was proposed, in which it balanced the load of each channel by constructing a node conflict graph, the adjacent vertices in the graph were sorted in descending order according to their load values, and then to allocate the channels. In [4], it proposed a maximum flow-based channel allocation algorithm (CMCA) using the maximum traffic flows to calculate the maximum throughput achieved in the network before channel assignment, and the traffic load of each link was used as the basis for channel allocation with minimum interference. However, this algorithm only considered the channel with the least interference in radio link with larger traffic load, but it ignored load balancing issue among the channels in their assignment. However, if the network traffic is heavy, some channels may be overloaded due to uneven traffic load among the channels, which may lead to network congestion and degrade system performance.
In this paper, we propose a maximizing capacity based network load-balancing channel allocation (MCLCA) algorithm which takes into account channel interference, actual channel capacity and link traffic load. A load balancing target optimization function is defined, a priority to the link with large traffic load is taken into account to allocate the channel with more the smallest channel usage rate so that the backbone links can have a larger traffic load to improve the whole network capacity.

\section{SYSTEM MODEL}

\section{A. Network Model}

We assumed that a WMN consists of $n$ wireless nodes, and the WMN is modeled as a connected graph $G=(V, E)$, where $V$ is the set of all node vertices and $E$ represents a set of all undirected edges in the WMN. It is assumed that each node $i \in$ $V$ configured with $R(i)$ network interface cards (NICs), and the orthogonal channel set is $C=\{1,2, \ldots, c\}$. For any two nodes $i, j \in V, d(i, j)$ represents the distance between nodes $i$ and $j$, and $r$ represents the transmission distance. If $d(i, j) \leq$ $r$, it means that nodes $i$ and $j$ are in their communication ranges. There is one undirected edge $e_{i j} \in E$ between nodes $i$ and $j$. The definitions of the parameters and functions in this paper are listed in Table 1.

\section{B. Interference Model}

The template is used to format your paper and style the text. All margins, column widths, line spaces, and text fonts are prescribed; please do not alter them. You may note peculiarities. For example, the head margin in this template measures proportionately more than is customary. This measurement and others are deliberate, using specifications that anticipate your paper as one part of the entire proceedings, and not as an independent document. Please do not revise any of the current designations. For the physical topology $G(V, E)$, if there are two edges $e_{i j} \in E, e_{m n} \in E$ and $\left.\min \{d(i, m), d(i, n), n)\right\} \leq$ $r^{\prime}$, where $r^{\prime}$ denotes the interference distance, then the two edges are said to be potential interference edges. For any link $e \in E, P I L(e)$ represents all potentially interfering link (PIL) sets of link $e, C(e)$ represents the channel allocated to link $e$, then all interfering links (IF) of link $e$ are set as $I F(e)=$ $\left\{e^{\prime} \mid e^{\prime} \in P I L(e), C(e)=C\left(e^{\prime}\right)\right\}$, where $e^{\prime} \in E$. 
Interference occurs when using the same channel for simultaneous communication in the same collision area. Therefore, during channel allocation, we let the links in the same conflict area use different channels. When the channel allocation for the links in the network is over, the channel used by each link is determined, then its set of interfering links is also known. At this point, for any link $e$, its interference can be expressed as

$$
I F(e)=\sum_{e^{\prime} \in P I L(e)} I\left(C\left(e^{\prime}\right)=C(e)\right), \forall e \in E, e^{\prime} \in E
$$

where $I\left(C\left(e^{\prime}\right)=C(e)\right)$ indicates that when the channel allocated on the potential interfering link $e^{\prime}$ is the same as the link $e$, it equals 1 , otherwise it equals 0 . Therefore, the interference of the entire network can be expressed as

$$
T I F=\sum_{e \in E} I F(e), \forall e \in E
$$

After channel assignment, a new communication topology $G c(V, E c)$ is completed, where $V$ also represents a set of all nodes in the WMN. Let $X(i)$ denote the set of channels assigned to node $i$, for any edge $e_{i j} \in E_{c}$ only if $e_{i j} \in$ $E, C(i) \cap C(j) \neq \Phi X$, where $\Phi$ is a null set.

\section{Traffic Model}

A traffic model is built in this paper by referring link traffic model in [9]. First, assume that a service from source s node by ways of some intermediate nodes reaches the destination node $t$ with service rate $\sigma^{s t}$. Suppose the service is forwarded by link $e_{i j}$, we define a variable $L_{i j}^{s t}$ expressed as

$$
L_{i j}^{s t}=\left\{\begin{array}{cc}
1, & \sigma^{s t} \text { through link } e_{i j} \\
0, & \text { others }
\end{array}, \forall i, j, s, t \in V\right.
$$

It is assumed that the traffic load on this link is denoted by $L$, then the link load when the traffic from the source to the destination by way of link $e_{i j}$ can be expressed as

$$
L_{i j}^{s t}=\gamma_{i j}^{s t} \sigma^{s t}, \forall i, j, s, t \in V, e_{i j} \in E
$$

In the network, normally the traffic loads in up- and down links are not symmetrical $\gamma_{i j}^{s t} \neq \gamma_{j i}^{s t}$, so the load in link $e_{i j}$ is

$$
L_{i j}=\sum_{s, t \in V}\left(L_{i j}^{s t}+L_{i j}^{s t}\right), \forall i, j, s, t \in V, e_{i j} \in E
$$

Since the number of orthogonal channels is much less than the number of node radios, some of the network links will work on the same channel. At this point, if they are in the same interference zone, interference will occur during simultaneous communications, and the actual link capacity will be decreased, which depends on the number of links using the same channels. Therefore, the actual capacity of link $e_{i j}$ can be defined as

$$
\begin{aligned}
c_{i j} & =\frac{B}{1+\sum_{e \in P I L\left(e_{i j}\right)} I\left(C(e)=C\left(e_{i j}\right)\right)} \\
& =\frac{B}{1+I F\left(e_{i j}\right)}, \quad \forall i, j \in V, e_{i j} \in E
\end{aligned}
$$

where $\mathrm{B}$ is the channel intrinsic capacity of $e_{i j}$, and $c_{i j}$ is the realistic channel capacity. Meanwhile, $\mu_{i j}$ is used to indicate the load ratio of link $e_{i j}$, which is the ratio of its service load to its actual link capacity,

$$
\mu_{i j}=\frac{L_{i j}}{c_{i j}}=\frac{L_{i j}}{B}\left(1+I F\left(e_{i j}\right)\right), \forall \mathrm{i}, \mathrm{j} \in \mathrm{V}, e_{i j} \in E
$$

when we allocate a channel to the link in addition to satisfying the constraints of the channel assignment algorithm, it should be ensured that the set of link traffic flows can be scheduled. If $L_{i j}$ indicates the traffic load of link $e_{i j}$, after a time interval $T$, the traffic transmitted by this link is $L_{i j} T$. If the capacity of link $e_{i j}$ is $c_{i j}$, then it takes $\left(L_{i j} / c_{i j}\right)^{*} \mathrm{~T}$ time to transmit $L_{i j} T$ amount of traffic. For any link $e_{i j}$, the data transmission time does not exceed $T$ in case of collision, namely $\sum_{e_{i j} \in I F(e)} \frac{L_{i j}}{c_{i j}} T \leq T$, the links are then called schedulable. Because $\mu_{i j}=\frac{L_{i j}}{c_{i j}}$, the inequality can be also expressed as

$$
U(e)=\sum_{e_{i j} \in I F(e)} \mu_{i j} \leq 1, \forall e \in E
$$

\section{Channel Assignment SCHEME}

\section{A. Algorithm Target}

From eqn. (7), we know that $\mu_{i j}$ is proportional to load $L_{i j}$ and interference level $I F\left(e_{i j}\right)$ for link $e_{i j}$. If $\mu_{i j} \leq 1$, it means that the link capacity can meet its traffic load requirement, smaller $\mu_{i j}$ indicates that the link has more remaining bandwidth to admit new requested services. That is, the goal of load balancing is to minimize $\mu_{i j}, \forall i, j \in V, e_{i j} \in E$. In the entire channel allocation procedure, the MCLCA algorithm optimizes the links with the maximum load in the network to ensure that the capacity of channel bandwidth allocation on the links can meet its service. Therefore, the objective function of MCLCA algorithm is: $\min \left(\max _{e \in E} \sum_{e_{i j} \in I F(e)} \mu_{i j}\right), \forall i, j \in$ $V, e_{i j} \in E$.

\section{B. Algorithm Description}

As aforementioned, implementation of MCLCA algorithm can be divided into two phases. In the first-phase, for any node $i \in V$, assume that neighbor $(i)$ represents the set of all neighbor nodes of $i$, and $\zeta$ denotes the set of all the logic groups for related nodes and links. $\zeta(i)$ denotes the logic link group for node $i, g$ represents the group number. In each round of iterations, there will be a node assigned to the corresponding group. Then, in the second phase, the set of related nodes which belong to group $g$ is denoted $N(g) . \rho_{c}$ represents usage rate of channel $c \in C$.

The algorithm in the first phase is described as follows:

Step 1: Sort the links $e_{i j}(j \in$ neighbor $(i))$ for node $i$ in $G(V, E)$ in descending order its the traffic flows, and then group them in sequence so that the number of traffic flows per node is not larger than its radio interfaces.

Step 2: Group the links $e_{i j}$. 
1) Assign a new group $g(g \in \zeta)$ to the link $e_{i j}$ if $\zeta(i)<$ $R(i), \zeta(j)<R(j)$.

2) Otherwise, select a group $g \in(\zeta(i) \cup \zeta(j))$ and calculate its $\mu_{i j}$, and assign the link with the smallest $\mu_{i j}$ to group $g$.

Step 3 Group all the links by Step 2. The priority to select and allocate group to link $e_{i j}$ is to minimize $\mu_{i j}$. The constraints for achieving minimum $\mu_{i j}$ are

$$
\begin{gathered}
\sum_{g \in \zeta} x_{i, g} \leq R(i) \quad \forall i \in V \\
\sum_{g \in \zeta} y_{e, g}=1 \quad \forall e \in E \\
x_{i, g}+x_{j, g} \geq 2 y_{e, g} \quad \forall e=i \leftrightarrow j \in E, g \in \zeta \\
\mu_{i j} \leq 1 \quad \forall i, j \in V \\
\mu_{i j}=\frac{L_{i j}}{B}\left(1+\operatorname{IF}\left(e_{i j}\right)\right) \quad \forall i, j \in V, \forall e \in E
\end{gathered}
$$

In (11)-(15), the binary variable $x_{i, g}$ represents the constraints between node i and group $g$, and $y_{e, g}$ represents the constraints between link e and group $g, x_{i, g}, y_{e, g} \in\{0,1\}$. When $x_{i, g}$ or $y_{e, g}$ equals 1 , it means node $i$ or link $e$ has allocated to the same group $g$. Otherwise, it indicates there is no channel allocated between these nodes. Eq. (11) indicates that the number of groups assigned to node $i$ is not greater than the number of its radio interfaces. Eq. (12) indicates that any link $e$ can only belong to one specific group. Eq. (13) means only if the two terminal nodes are assigned to group $g$ at the same time, then its link belongs to group $g$ as well. Eq. (14) indicates that the link capacity satisfies the required service bandwidth restrictions.

The second phase of the algorithm is as follows:

Step 1 Calculate the load rates of all the links in their interference domains, which is to calculate $U(e)=$ $\sum_{e_{i j \in I F(e)}} \mu_{i j}$, and sort the links with the maximum load rate for each group in descending order, and then perform channel allocation in turn.

Step 2 If the maximum link load rate in group $g$ is also the maximum link load rate in all the current groups, the channels with the smallest channel usage rate $\rho_{c}$ are selected to be allocated to the links in group $g$, and then the selected channel result is sent to other groups. Otherwise, the terminals wait for receiving channel selection information in the network, and will participate in the next round of channel allocation.

Step 3 Repeat step 2, assign the channels group by group until all the links have remaining channels to communicate.

\section{SimULATION RESULTS}

In this paper, we use NS2 [5] to implement simulation. The network topology is shown in Fig.1, in which we have 40 nodes randomly distributed in a square with size of $1500 \mathrm{~m} \times 1500 \mathrm{~m}$. Assume that the transmission and interference distances are 250m and 500m, respectively. By using our proposed MCLCA algorithm, the radio interfaces and channels are assigned as show in Figure 1.

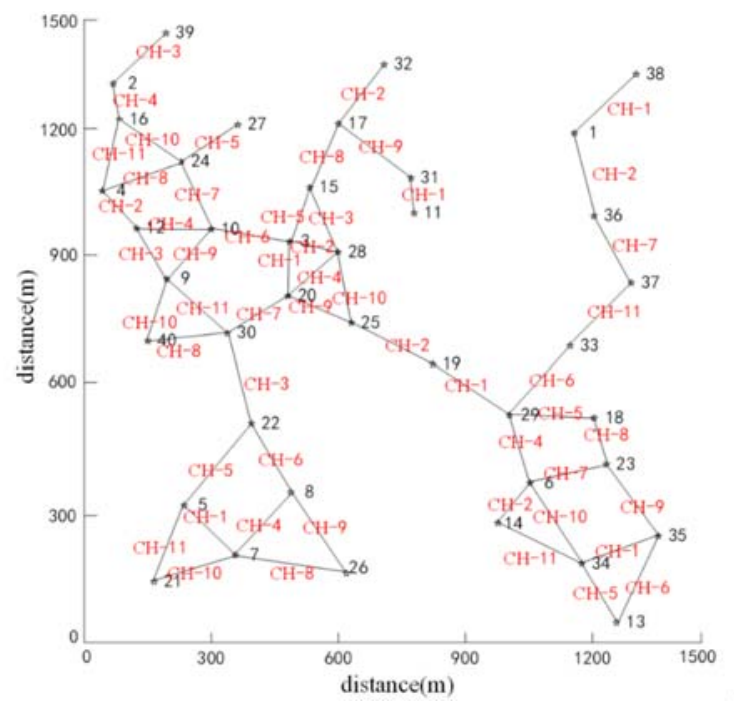

FIGURE I. NETWORK TOPOLOGY AND MULTI-RADIO MULTICHANNEL ALLOCATION SCHEME

In simulations, we assume that the maximum radio interfaces in a node is 4, and there are 11 non-overlapping channels in the network. The traffic flows are generated randomly and increased between the network nodes. The traffic flows are assumed as CBR (constant bit rate) with rate of 500 $\mathrm{kbit} / \mathrm{s}$, and the simulation time is 10 seconds. The key performance indicators (KPI) under simulation are the network throughput, end-to-end delay, and packet loss rate. With the increased number of traffic flows, the KPIs of the CMCA, LBCA and MCLCA algorithms are simulated and compared as shown in Figure 2 - Figure 4.

Figure 2 shows network throughputs with different traffic loads. It can be seen that at the beginning, the three algorithms have small difference in throughput. As the number of traffic flows are increased, the CMCA throughput is better than the other two algorithms with light traffic load. However, when the number of traffic flows is more than 15, our proposed MCLCA algorithm has the best performance, and the LBCA is better CMCA algorithm. Because the MCLCA algorithm is a load balancing channel allocation algorithm, which first groups packets according to link load, and balances the traffic load for each group to reduce its traffic load among the groups. Finally, according to the load of the links, different priorities in their channel allocation are given to achieve the maximum network capacity. Therefore, the MCLCA algorithm has a larger network capacity than the other two algorithms when network traffic load is heavy. 


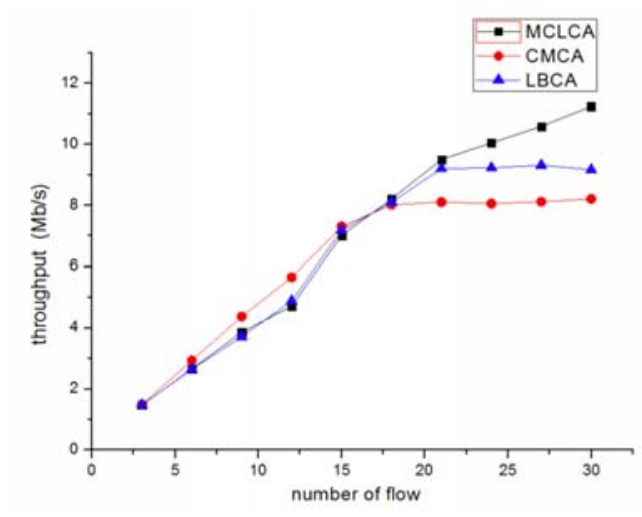

FIGURE II. THROUGHPUT COMPARISONS OF THE THREE ALGORITHM WITH THE NUMBER OF TRAFFIC FLOWS

Figure 3 shows comparisons of the end-to-end delays of the three algorithms in different network traffic load. Overall, the delays are increased with the number of traffic flows. When network load is light, the delays of the three algorithms are roughly the same. With the increasing number of data flows, the CMCA delays are increased rapidly, while the MCLCA and LBCA delays are increased relatively less. This is because the CMCA algorithm aims to minimize network interference, however, when network load is heavy, the CMCA algorithm cannot adapt to the changes of network traffic. The transmission delays are increased rapidly with worse network performance at the same time.

Figure 4 shows packet loss rate with respect to network traffic load. From the simulation results we can conclude that, the packet loss rates of the three algorithms increase with the number of traffic flows. When traffic load is light, they all have small packet loss rates, meanwhile the CMCA algorithm has a lower packet loss ratio because of less inter-channel interference. When the number of traffic flows increases, the packet loss rate of the CMCA algorithm increases more rapidly because the it lacks load balancing mechanism. At the same time, compared with LBCA algorithm, MCLCA considers the remaining channel bandwidth and load balance, which can achieve smaller packet loss rate.

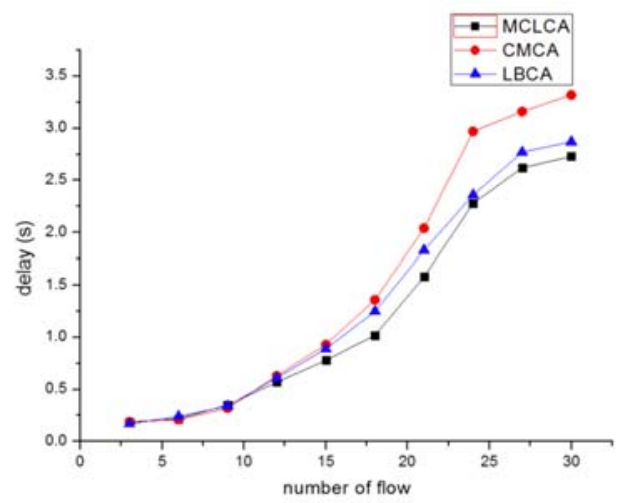

FIGURE III. END-TO-END DELAY COMPARISONS OF THE THREE ALGORITHMS WITH THE NUMBER OF TRAFFIC FLOWS

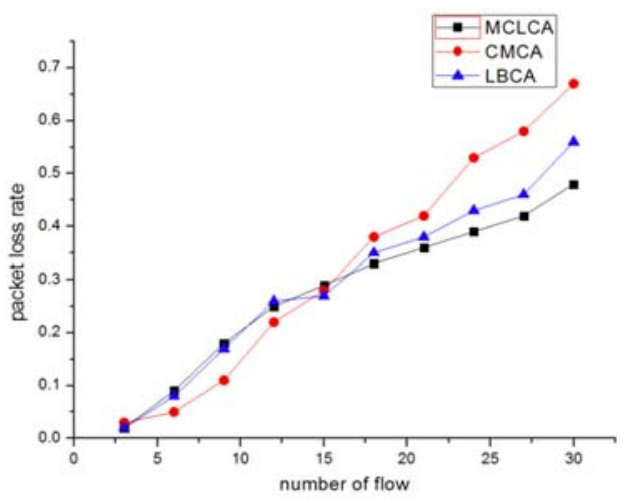

FIGURE IV. PACKET LOSS RATE COMPARISONS OF THE THREE ALGORITHMS WITH THE NUMBER OF TRAFFIC FLOWS

\section{CONCLUSION}

In this paper, a load balancing channel allocation algorithm is proposed by maximizing network capacity, in which the load balancing among channels in a wireless mesh network in considered to reduce end-to-end delay and packet loss rate for better network QoS. The channel assignment algorithm is implemented into two phases, the first phase is to group all the links in a mesh network, then to balance the traffic load in each group and find the load difference in each group. In the second phase, we allocate the same channel with the smallest channel usage rate to the links with larger load ratios in the groups, which can reduce interference and avoid repeated recursive iterations of the algorithm due to the restriction of the number of node interfaces during channel assignment to improve channel allocation efficiency. The simulation results show that our proposed algorithm can balance network traffic load well to achieve better network performance, especially it can improve network capacity with heavy traffic load.

\section{ACKNOWLEDGMENT}

This work was supported by the Department of Science and Technology, State Grid, China under contract no. SGSDJY00 GPJS1600298.

\section{REFERENCES}

[1] A. B. M. Alim Al Islam, M. J. Islam, N. Nurain and V. Raghunathan, "Channel Assignment Techniques for Multi-Radio Wireless Mesh Networks: A Survey," IEEE Communications Surveys \& Tutorials, vol. 18, no.2, pp. 988-1017, Secondquarter 2016.

[2] Y. Li, P. Wu and X. Liu, "Capacity-Based Channel Assignment Scheme in Multi-radio Multi-channel Wireless Mesh Networks,” Chinese Journal of Electronics, vol.24, no.2, 2016,pp. 419-425.

[3] W. W. Zhang, J. F. He, K. Bing and X. J. Shen, ”Research on channel assignment algorithm in hybrid wireless mesh networks," Journal of Chinese Computer Systems, vol.30, no.5,2009,pp. 812-816.

[4] Z. H. Ge, T. S. Li and Y. H. Wei., "A new max-flow based channel assignment algorithm for wireless mesh network," Telecommunications Science,vol.27,no.12,2011,pp. 53-56.

[5] V. Y. Deshmukh, P. S. Varade and Y. Ravinder, "Channel selection strategy for Cognitive Radio using NS2," International Conference on Human Computer Interactions, 2013,pp. 1-5. 